Dans quels pays les enseignants les plus qualifiés et expérimentés exercent-ils dans les établissements les plus difficiles?

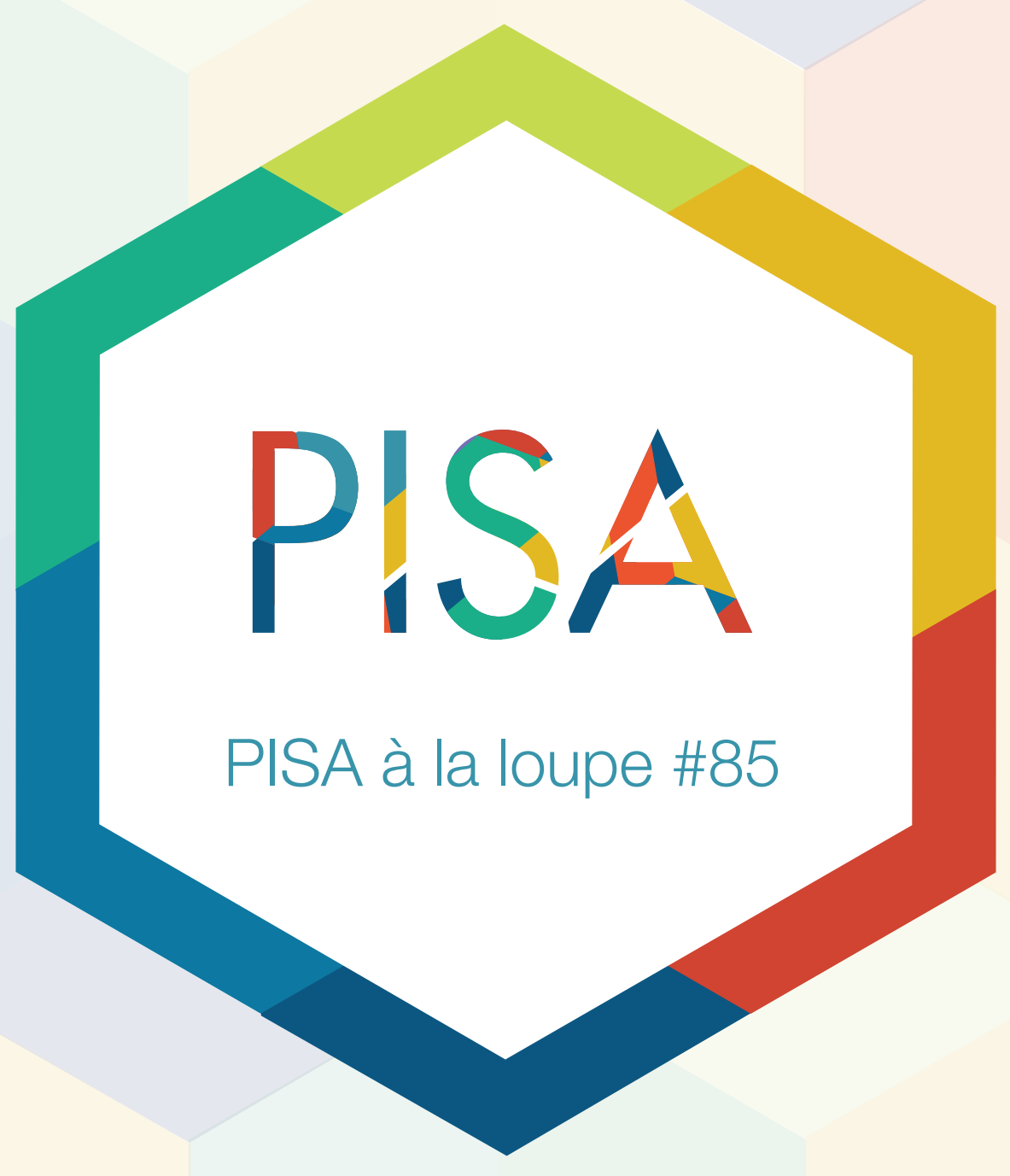




\section{Dans quels pays les enseignants les plus qualifiés et expérimentés exercent-ils dans les établissements les plus difficiles ?}

- D'après les données de 2015, la majorité des pays et économies participant à l'enquête PISA compensent les difficultés des établissements défavorisés par une réduction de la taille des classes et/ou une augmentation du taux d'encadrement. Toutefois, dans plus d'un tiers des pays et économies, les enseignants des établissements les plus défavorisés sont moins qualifiés ou moins expérimentés que ceux des établissements les plus favorisés.

- Les écarts de performance entre les élèves en relation avec leur statut socio-économique sont plus marqués dans les pays où les établissements défavorisés sur le plan socio-économique emploient moins d'enseignants qualifiés et expérimentés que les établissements favorisés.

- Une plus grande autonomie des établissements au regard de la gestion des enseignants est associée à une affectation plus équitable de ces derniers entre les établissements.

Les enseignants constituent la ressource scolaire principale. Dans tous les pays, leur salaire et leur formation représentent la part la plus importante des dépenses d'éducation - un investissement par ailleurs susceptible d'avoir des rendements considérables. Les travaux de recherche montrent ainsi que le fait de recevoir leur instruction des meilleurs enseignants peut avoir une réelle incidence sur la réussite scolaire et personnelle d'élèves par ailleurs similaires.

Toutefois, tous les élèves ne sont pas égaux en termes d'accès à un enseignement de qualité. Les données de l'enquête PISA révèlent ainsi l'existence, dans de nombreux pays, d'inégalités d'accès à des enseignants expérimentés et qualifiés, ainsi que la relation de ces inégalités avec les écarts de résultats d'apprentissage entre élèves favorisés et défavorisés.

\section{Quantité ne rime pas nécessairement avec qualité.}

Une analyse des données de l'enquête PISA 2015 a permis de classer l'ensemble des établissements des premier et deuxième cycles de l'enseignement secondaire (selon le niveau d'enseignement typiquement fréquenté par les jeunes de 15 ans) en fonction de leur profil socio-économique - soit le statut socio-économique moyen de l'effectif d'élèves de 15 ans de l'établissement. Une fois ce classement effectué, les établissements sont répartis en quatre groupes dans chaque pays, chacun regroupant environ $25 \%$ de l'effectif total d'élèves de 15 ans. Les groupes d'établissements ayant le profil socio-économique moyen le plus faible/le plus élevé représentent respectivement les établissements défavorisés/favorisés sur le plan socio-économique. L'analyse s'attache ensuite à comparer, entre établissements favorisés et défavorisés, la taille des classes, les taux d'encadrement, les qualifications des enseignants et, pour les pays ayant collecté ces données, leur expérience professionnelle.

Les résultats montrent clairement que la majorité des pays et économies ayant participé à l'enquête PISA 2015 compensent les difficultés des établissements défavorisés par une réduction de la taille des classes et/ou une augmentation du taux d'encadrement ; ce constat vaut particulièrement lorsque l'analyse se limite aux établissements publics ou privés subventionnés par l'État. Toutefois, dans plus d'un tiers des pays et économies - y compris un certain nombre de ceux qui compensent les difficultés des établissements défavorisés par l'étoffement de leur personnel enseignant -, les enseignants des établissements les plus défavorisés sont moins qualifiés et/ou expérimentés que ceux des établissements les plus favorisés.

L'effectif d'élèves des classes de la langue d'enseignement est plus réduit dans les établissements défavorisés que dans les établissements favorisés dans 38 des 69 pays et économies participant à l'enquête PISA, notamment en Allemagne, au Canada, en Corée, en France, au Japon, au Mexique, aux Pays-Bas et en Pologne. Parallèlement, même si la taille des classes n'y diffère pas de façon significative entre établissements défavorisés et favorisés, le Danemark, l'Espagne, l'Irlande et l'Italie, ainsi que les États-Unis pour les établissements publics et privés subventionnés par l'État, compensent les difficultés des établissements défavorisés par l'augmentation du taux d'encadrement.

Cependant, les systèmes d'éducation sont bien moins nombreux à veiller à ce que les enseignants plus qualifiés ou une part plus importante d'enseignants pleinement certifiés exercent dans les établissements défavorisés. De fait, c'est souvent l'inverse qui 
Différence de ressources en personnel enseignant entre les établissements favorisés et défavorisés Résultats fondés sur les déclarations des chefs d'établissement

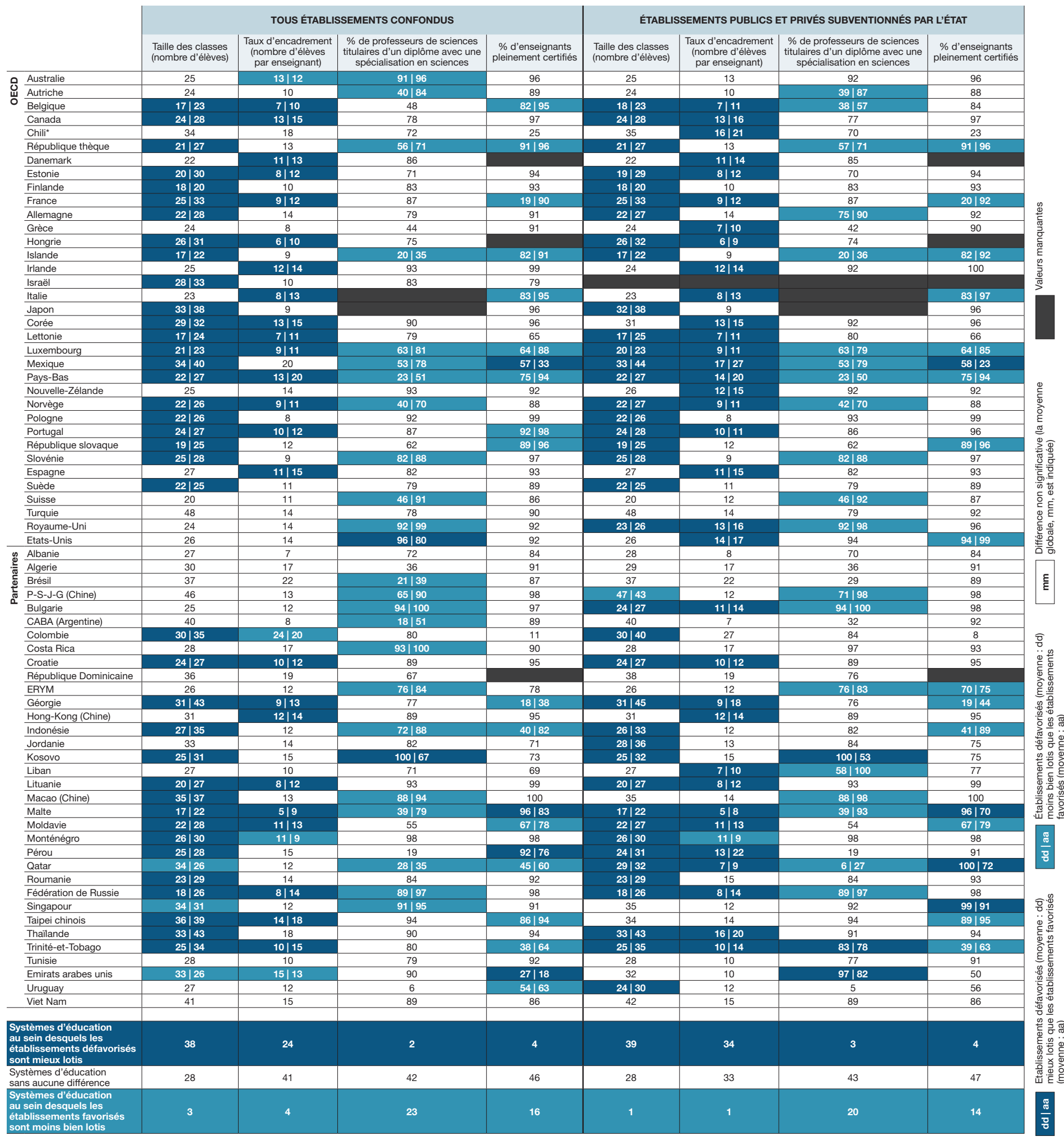

*Au Chili, la question relative aux qualifications des enseignants a été adaptée comme suit : " agréés ou habilités par le ministère de l'Éducation ".

Remarques : Les différences de taille des classes de moins de deux élèves et de taux d'encadrement de moins d'un élève ne sont pas considérées comme significatives ; les différences de pourcentage de professeurs de sciences titulaires d'un diplôme avec une spécialisation en sciences et de professeurs pleinement certifiés de moins de 4 points de pourcentage ne sont pas considérées comme significatives. Les différences plus importantes sont signalées comme significatives sur la base de l'estimation des erreurs-types.

Les pays et économies sont classés en fonction de leur statut (membre ou partenaire de l'OCDE) et par ordre alphabétique de leur nom anglais.

Source : OECD (2018), Effective Teacher Policies: Insights from PISA, Figure 1.2. 


\section{Relation entre les différences socio-économiques de performance} en compréhension de l'écrit et de taille des classes

Différence de performance en compréhension de l'écrit entre les élèves du quartile supérieur du statut socio-économique et ceux du quartile inférieur, et différence moyenne de taille des classes de la langue d'enseignement entre les établissements favorisés et défavorisés

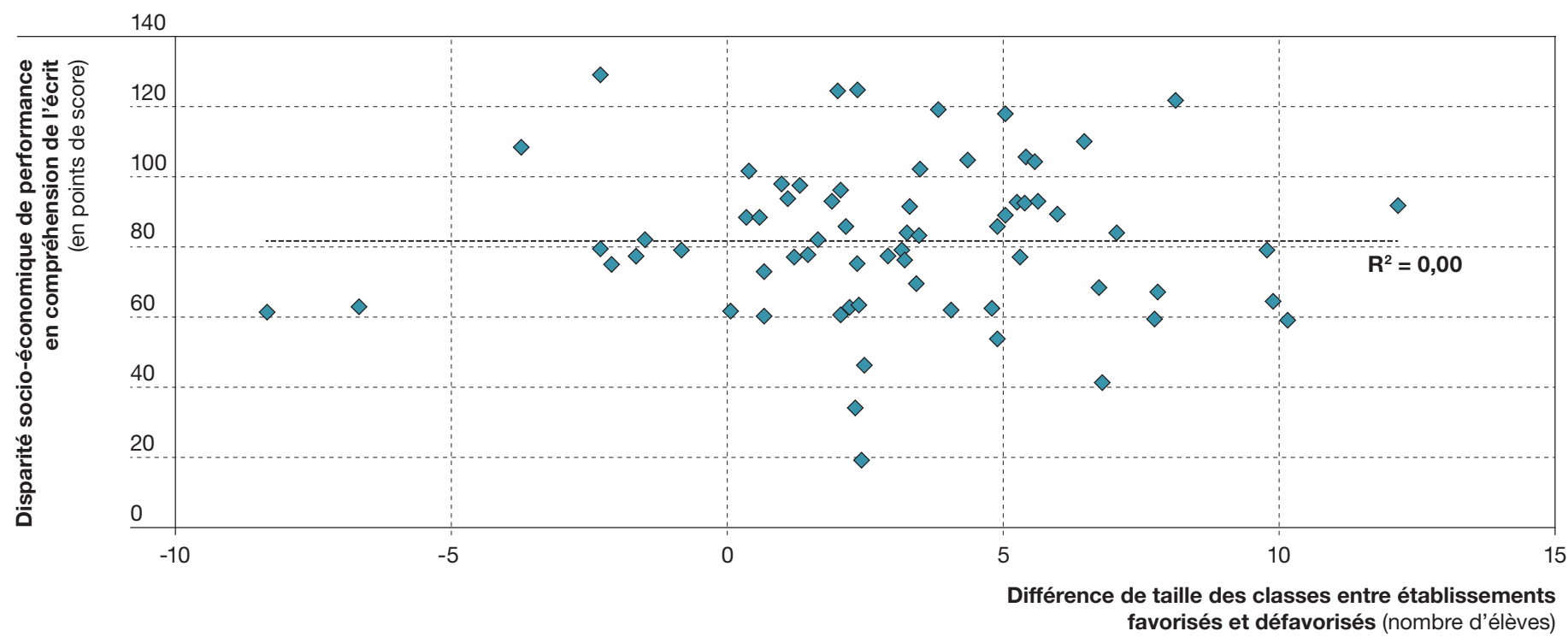

Remarque : La ligne en pointillé indique une relation non significative.

Source : OCDE (2018), Effective Teacher Policies: Insights from PISA, tableau 3.1, OCDE (2016), Résultats du PISA 2015 (Volume l) : L'excellence et l'équité dans l'éducation, tableau I.6.3b, http://dx.doi.org/10.1787/888933433214

s'observe. En France, en Italie et aux Pays-Bas, et dans 16 des 66 pays/économies à l'étude, les enseignants des établissements les plus défavorisés sont moins susceptibles d'être pleinement certifiés que ceux des établissements favorisés ; un écart similaire s'observe entre établissements publics favorisés et défavorisés aux États-Unis. En Australie, au Mexique et au Royaume-Uni, et dans 23 des 67 pays/économies à l'étude, les professeurs de sciences des établissements les plus défavorisés sont moins susceptibles d'être titulaires d'un diplôme universitaire avec une spécialisation en sciences que leurs homologues des établissements favorisés. Dans les pays ayant administré un questionnaire spécifique aux enseignants dans le cadre de l'enquête PISA 2015, des écarts similaires - au détriment des établissements défavorisés - s'observent également pour d'autres caractéristiques des enseignants corrélées à leur qualité, telles que le pourcentage d'enseignants ayant plus de 5 ans d'exercice dans la profession ou de ceux travaillant sous contrat à durée déterminée d'une durée de moins d'un an.

\section{L'éducation doit s'efforcer de remédier aux inégalités économiques et sociales, et non les accentuer.}

Si des disparités de performance entre les élèves en relation avec leur statut socio-économique s'observent dans tous les pays, ceux où les qualifications et l'expérience des enseignants sont d'un niveau significativement plus élevé dans les établissements favorisés que dans les établissements défavorisés tendent à présenter des écarts de performance plus marqués selon le statut socio-économique des élèves, et donc des résultats moins équitables. En revanche, les pays qui compensent le désavantage de certains établissements par une réduction de la taille des classes et une augmentation du taux d'encadrement ne présentent pas, en moyenne, des écarts de performance plus réduits entre les différents statuts socio-économiques. Ce constat pourrait s'expliquer par le fait que ce type de compensations quantitatives ne rime pas nécessairement avec l'accès à des enseignants et à un enseignement de meilleure qualité. II semble en outre indiquer qu'il n'est pas suffisant, voire pas nécessaire, pour les établissements les plus défavorisés de disposer de davantage d'enseignants, tant qu'ils sont en mesure d'attirer les professionnels les plus talentueux et efficaces.

Les adversaires de l'autonomisation des établissements font souvent valoir que le renforcement de l'indépendance des établissements pourrait entraîner de plus fortes disparités sur le plan de la performance des élèves et - argument peut-être encore plus préoccupant 


\section{Relation entre les différences socio-économiques de performance en sciences et de qualification des enseignants}

Différence de performance en sciences entre les élèves du quartile supérieur du statut socio-économique et ceux du quartile inférieur, et différence moyenne de pourcentage de professeurs de sciences titulaires d'un diplôme avec une spécialisation en sciences entre les établissements favorisés et défavorisés

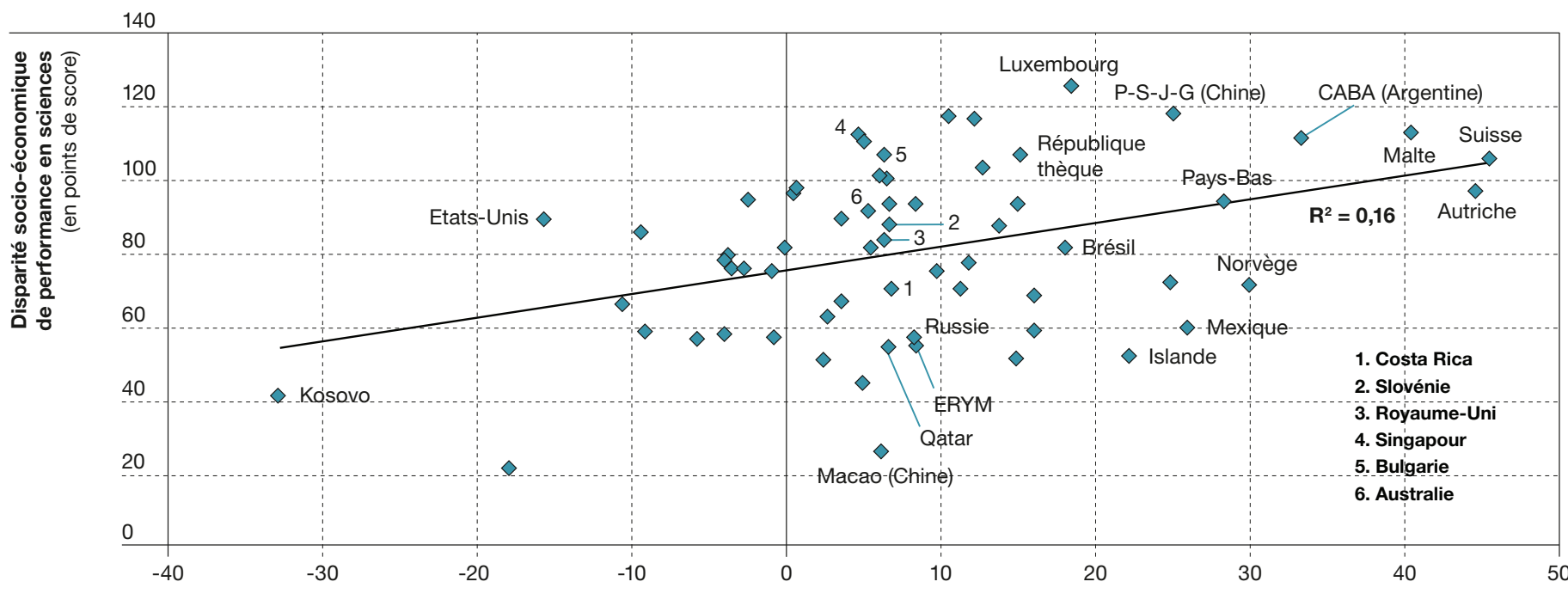

Différence de qualification des enseignants entre établissements favorisés et défavorisés (en points de pourcentage)

Remarque : Dans les pays présentés dans ce graphique, une différence significative s'observe entre les établissements favorisés et défavorisés dans le pourcentage de professeurs de sciences titulaires d'un diplôme avec une spécialisation en sciences. Les pays/économies où cette différence n'est pas significative sont les suivants : Albanie, Algérie, Allemagne, Belgique, Canada, Chili, Colombie, Corée, Danemark, Émirats arabes unis, Espagne, Estonie, Finlande, France, Géorgie, Grèce, Hong-Kong (Chine), Hongrie, Indonésie, Irlande, Israël, Jordanie, Lettonie, Liban, Lituanie, Moldavie, Monténégro, Nouvelle-Zélande, Pérou, Pologne, Portugal, République dominicaine, République slovaque, Roumanie, Suède, Taipei chinois, Thaillande, Trinité-et-Tobago, Tunisie, Turquie, Uruguay et Viet Nam. Source : OCDE (2018), Effective Teacher Policies: Insights from PISA, tableau 3.11; OCDE (2016), Résultats du PISA 2015 Nolume I) : L'excellence et l'équité dans l'éducation, tableau I.6.3a, http://dx.doi.org/10.1787/888933433214

- pourrait mener à un système d'éducation qui accentue les inégalités socio-économiques existantes au lieu d'y remédier. Toutefois, les données de l'enquête PISA semblent indiquer qu'il ne s'agit pas là de la conséquence la plus courante du renforcement de l'autonomie des établissements. De nombreux pays ont su combiner l'octroi d'une grande autonomie à leurs établissements à de fortes incitations afin de garantir que les établissements privilégient l'apprentissage des élèves par rapport à d'autres considérations, et à des mécanismes de financement compensatoires visant à veiller au maintien de l'équité. L'Irlande en offre un exemple intéressant : alors que la plupart des établissements y sont privés, les pouvoirs publics assurent la quasi-totalité du financement; en outre, les établissements irlandais accueillant les concentrations les plus fortes d'élèves issus de milieux défavorisés reçoivent des financements significativement plus importants et bénéficient d'un accès prioritaire aux programmes éducatifs gouvernementaux, notamment aux programmes de formation des enseignants. C'est peut-être la raison pour laquelle, par rapport aux établissements favorisés, les établissements les plus défavorisés en Irlande proposent non seulement un taux d'encadrement plus élevé, mais aussi des enseignants au moins aussi qualifiés.

\section{Pour conclure}

La plupart des pays peuvent encore progresser en termes de supervision de l'affectation des enseignants entre les différents établissements : il ne s'agit pas uniquement de contrôler leur nombre, mais aussi d'attacher une importance toute particulière à leurs qualifications, leur expérience et leur efficacité. Toute politique concernant les enseignants et visant à pallier le désavantage des élèves doit s'efforcer de veiller à la qualité, et pas seulement à la quantité, des enseignants affectés auprès des élèves défavorisés. 


\section{Pour tout complément d'information}

Contacter : Francesco Avvisati (francesco.avvisati@oecd.org)

Consulter : OCDE (2018), Effective Teacher Policies : Insights from PISA, PISA, Éditions OCDE, Paris, http://dx.doi.org/10.1787/9789264301603-en.

Le mois prochain : Quel lien entre les pratiques sportives des élèves et leur performance et leur bien-être?

Cet ouvrage est publié sous la responsabilité du Secrétaire général de l'OCDE. Les opinions et les interprétations exprimées ne reflètent pas nécessairement les avis des pays membres de l'OCDE.

Ce document, ainsi que les données et cartes qu'il peut comprendre, sont sans préjudice du statut de tout territoire, de la souveraineté s'exerçant sur ce dernier, du tracé des frontières et limites internationales, et du nom de tout territoire, ville ou région.

Les données statistiques concernant Israël sont fournies par et sous la responsabilité des autorités israéliennes compétentes. L'utilisation de ces données par l'OCDE est sans préjudice du statut des hauteurs du Golan, de Jérusalem Est et des colonies de peuplement israéliennes en Cisjordanie aux termes du droit international.

Ce texte est disponible sous licence Attribution - Pas d'Utilisation Commerciale - Partage dans les Mêmes Conditions 3.0 Organisations Internationales (CC BY-NC-SA 3.0 IGO). Pour toute information spécifique quant à l'étendue et aux termes de la licence ainsi que d'une possible utilisation commercial de ce texte et pour toute usage de données PISA, prière de consulter les Conditions d'utilisation à http://www.oecd.org/fr/conditionsdutilisation. 